\title{
Statistical model for fading return signals in coherent lidars
}

\author{
Aniceto Belmonte \\ Department of Signal Theory and Communications, Technical University of Catalonia, \\ BarcelonaTech, 08034 Barcelona, Spain (belmonte@tsc.upc.edu) \\ Received 21 July 2010; accepted 24 October 2010; \\ posted 27 October 2010 (Doc. ID 131960); published 6 December 2010
}

\begin{abstract}
A statistical model for the return signal in a coherent lidar is derived from the fundamental principles of atmospheric scattering and turbulent propagation. The model results in a three-parameter probability distribution for the coherent signal-to-noise ratio in the presence of atmospheric turbulence and affected by target speckle. We consider the effects of amplitude and phase fluctuations, in addition to local oscillator shot noise, for both passive receivers and those employing active modal compensation of wavefront phase distortion. We obtain exact expressions for statistical moments for lidar fading and evaluate the impact of various parameters, including the ratio of receiver aperture diameter to the wavefront coherence diameter, the speckle effective area, and the number of modes compensated. (C) 2010 Optical Society of America
\end{abstract}

OCIS codes: $\quad 010.3640,010.1330,010.1290$.

\section{Introduction}

During the past few decades, coherent lidar has established itself as a unique instrument in atmospheric remote sensing and its application area has broadened considerably (see, e.g., [11-[10]) along with the technologies contributing to it [11]. Coherent lidars are currently employed in a large variety of atmospheric applications in fields as diverse as laser vibrometry and target identification [1,2], meteorological observation and atmospheric wind determination [3, 目, aerosol and atmospheric constituent concentration measurements [5-7], and tracking and control of pollutants atmospheric fluxes [8-10].

Many coherent lidar applications impose stringent power constraints while requiring high levels of sensitivity and accuracy. Therefore, to optimize the lidar system parameters and ensure full utilization of limited resources, it is of paramount importance to have a clear understanding of all the possible sources of external disturbances, i.e., target speckle and atmospheric refractive turbulence, affecting lidar measurements. It is, then, natural that a problem of

$0003-6935 / 10 / 356737-12 \$ 15.00 / 0$

(C) 2010 Optical Society of America continuing interest among researchers studying coherent lidar concerns the definition of a model for the physical mechanisms involved in the lidar problem and the statistical form of the lidar signal fluctuations (see, e.g., [12 18]).

Evaluating the performance of a heterodyne or homodyne lidar receiver in the presence of atmospheric turbulence and target speckle is generally difficult because of the complex ways turbulence and speckle affect the coherence of the received signal that is to be mixed with the local oscillator. The downconverted heterodyne or homodyne power is maximized when the spatial field of the received signal matches that of the local oscillator [12]. Any mismatch of the amplitudes and phases of the two fields will result in a loss in downconverted power, i.e., fading. Here we study, in a unified framework, the effects of both wavefront distortion and amplitude scintillation, in addition to local oscillator shot noise, for both passive receivers and those employing active modal compensation of wavefront phase distortion. Adaptive compensation of atmospheric wavefront phase distortion to improve the performance of atmospheric systems has been an important field of study for many years. In particular, the modal compensation method involves correction of several modes of 
an expansion of the total phase distortion in a set of basis functions.

In lidar systems, signal fluctuations could result from physical mechanisms other than target speckle, primarily refractive turbulence, so these mechanisms must also be the focus of this analysis. In general, fluctuations induced by turbulence are not as intense as those due to speckle in optical remote sensing systems but, although their normalized variance is smaller, they still need to be considered to properly describe the performance of any practical coherent lidar. Much work has been published on coherent lidar theory. Early analytical work [12-17] on the problem concentrated on the reduction of heterodyne system efficiency caused by beam distortion under limited conditions that provide a reliable basis for preliminary assessment of lidar performance. These mostly heuristic analyses statistically quantified turbulence-induced fading through its mean and variance, although they alone are not adequate to fully characterize system performance. Later analyses used in heterodyne lidar [18] attempted to overcome these limitations and fully characterize the statistics of heterodyne optical systems by defining theoretical expressions based on the path-integrated technique (see, e.g., [19]), one of the theoretical asymptotic methods for estimating the higher moments of the fields propagated through random media. This technique predicts the leading-order effects of refractive turbulence with minimal approximations, providing a framework valid for any typical path-integrated atmospheric refractive turbulence. However, theoretical calculation of beam propagation and the higher moments of the field is still difficult and, consequently, no simple analytical solutions are known beyond those obtained for simplified beam configurations and unrealistic atmospheric characterization. More recently, full-wave simulation of beam propagation has been used to examine the uncertainty inherent to the process of lidar heterodyne optical power measurement because of the presence of refractive turbulence for both passive receivers [20] and those using modal compensation of wavefront phase distortion [21]. With the two-beam model, the lidar return is expressed in terms of the overlap integral of the transmitter and virtual (backpropagated) local oscillator beams at the target, reducing the problem to one of computing irradiance along the two propagation paths. Although the simulation of beam propagation permits the full statistical examination of the signal degradation in a heterodyne receiver caused by refractive turbulence under general atmospheric conditions and at arbitrary transmitter and receiver configurations, the technique is computationally demanding.

In this work, our formulation circumvents the need for a detailed description of the compound speckle and turbulence problem. Such a specification is difficult because of the inherent complexity of the propagation, as well as the random distribution of the atmospheric scatters. Instead, our phenomenological model first replaces the turbulence-distorted wavefront by an equivalent speckle representation of randomly dispersed coherent contributions. It then compounds the resulting turbulence fading statistics with target speckle statistics. The generalized $K$ distribution proposed in this study to describe fading return signals in heterodyne lidar receivers was derived under the assumption that the average coherent signal is a random variable driven by atmospheric turbulence. In this situation, the mean of the target speckle signal is smeared by turbulence speckle fluctuations, and we need to define multiply stochastic (compound) statistics to describe the return signals in a coherent lidar.

In Section 2, after discussing the general properties of speckle and turbulence statistics, we proceed to develop the double stochastic representation of the coherent lidar return signals leading to the generalized $K$ distribution. In Section 3, we calculate and present the performance for both passive receivers and those employing active modal compensation of wavefront phase distortion. We consider the effects of amplitude and phase fluctuations. The conclusion is provided in Section $⿴$.

\section{Statistical Model for Coherent Fading Return Signals}

For a heterodyne receiver, with average power constraint $P$ and noise power spectral density $N_{0} / 2$, $\gamma_{0} P / N_{0} B$ is the signal-to-noise ratio (SNR) per unit bandwidth $B$. The SNR $\gamma_{0}$ for a quantum or shot-noise limited signal can be interpreted as the detected number of photons (photocounts) per pulse when $1 / B$ is the pulse period. Coherently detected signals are modeled as narrowband rf signals with additive white Gaussian noise (AWGN). For a heterodyne lidar system, in the presence of target speckle and atmospheric turbulence, we must consider fading signals, which are signals also affected by multiplicative noise. In the fading AWGN channel, we let $\alpha^{2}$ denote the atmospheric channel power fading and $\left(P / N_{0} B\right)$ $\alpha^{2}=\gamma_{0} \alpha^{2}$ denote the instantaneous received SNR per pulse. For a shot-noise-limited coherent optical receiver, the SNR of the envelope detector can be taken as the number of signal photons detected on the receiver aperture $\gamma_{0}$ multiplied by a heterodyne power mixing efficiency $\alpha^{2}$ : In addition to the effective delivery of the signal to the detector, the performance of the optical link also depends on the receiver sensitivity, measured in terms of received photons. For systems with perfect spatial mode matching, the mixing efficiency is equal to 1 . When the spatial modes are not properly matched, the contribution to the current signal from different parts of the receiver aperture can interfere destructively and result in reduced instantaneous heterodyne mixing and consequent fading. Note that, conditional on a realization of the atmospheric channel described by $\alpha^{2}$, this is an AWGN channel with instantaneous received SNR $\gamma=\gamma_{0} \alpha^{2}$. This quantity is a function of the random channel power fading $\alpha^{2}$ and is, therefore, random. The 
statistical properties of the atmospheric random channel fade $\alpha$, with mean-square value $\Omega=\overline{a^{2}}$ and probability density function (PDF) $P_{\alpha}(\alpha)$, provide a statistical characterization of the SNR $\gamma=\gamma_{0} \alpha^{2}$. In this study, we define a statistical model for the fading amplitude $\alpha$ (i.e., SNR $\gamma$ ) of the received signal scattered by the atmospheric target after propagation through the atmosphere.

When the spatial field of the received signal does not match that of the local oscillator, referred to the receiving aperture and assumed to be uniform there, the random channel fading

$$
\boldsymbol{\alpha}=\frac{4}{\pi D^{2}} \int \mathrm{d} \boldsymbol{r} W(\boldsymbol{r}) E_{S}(\boldsymbol{r})
$$

depends on amplitude and phase mismatches of the two fields incident on the receiving aperture through the random fluctuations $E_{S}(r)$ of a complex field pattern. $E_{S}(\boldsymbol{r})$ represents the amplitude fluctuations and phase distortions introduced by atmospheric turbulence and target speckle in the return signal. In general, fading is a complex magnitude $\boldsymbol{\alpha}=\alpha_{r}+$ $j \alpha_{i}$, where $\alpha_{r}$ and $\alpha_{i}$ represent integrals over the collecting aperture of the real and imaginary parts, respectively, of the normalized optical field reaching the receiver. These real and imaginary parts can be considered as the components of a complex random phasor. The circular receiving aperture of diameter $D$ is defined by the aperture function $W(r)$, which equals unity for $|r| \leq D / 2$, and equals zero for $|\boldsymbol{r}|>D / 2$. Note that $\boldsymbol{\alpha}$ has been normalized to be unity when there is a perfect match between both the received signal and the local oscillator, i.e., $E_{S}(\boldsymbol{r})=1$ for all $\boldsymbol{r}$. An explicit expression for the mean-square value $\Omega=\overline{a^{2}}$,

$$
\Omega=\overline{\boldsymbol{\alpha} \boldsymbol{\alpha}^{*}}=\left(\frac{4}{\pi D^{2}}\right)^{2} \overline{\int \mathrm{d} \boldsymbol{r} W(\boldsymbol{r}) E_{S}(\boldsymbol{r}) \int \mathrm{d} \boldsymbol{r}^{\prime} W\left(\boldsymbol{r}^{\prime}\right) E_{S}^{*}\left(\boldsymbol{r}^{\prime}\right),}
$$

can be found by writing the two integrals as a double integral and bringing the averaging operator inside the double integral:

$$
\Omega=\left(\frac{4}{\pi D^{2}}\right)^{2} \iint \mathrm{d} \boldsymbol{r} \mathrm{d} \boldsymbol{r}^{\prime} W(\boldsymbol{r}) W\left(\boldsymbol{r}^{\prime}\right) \overline{E_{S}(\boldsymbol{r}) E_{S}^{*}\left(\boldsymbol{r}^{\prime}\right)} .
$$

By changing the variables of integration from $r, r^{\prime}$ to $\boldsymbol{\rho}=\boldsymbol{r}-\boldsymbol{r}^{\prime}$ and $\boldsymbol{R}=\left(\boldsymbol{r}+\boldsymbol{r}^{\prime}\right) / 2$, and recognizing $\mu(\boldsymbol{\rho})=$ $E_{S}(\boldsymbol{R}+\rho / 2) E_{S}^{*}(\boldsymbol{R}-\rho / 2)$ as the spatial coherence function describing the average spatial correlation length of field fluctuations, we get

$$
\Omega=\frac{4}{\pi D^{2}} \int \mathrm{d} \rho K_{D}(\rho) \mu(\rho) .
$$

Here, $K_{D}(\rho)$ is the receiver circular aperture function, i.e., the fractional area of two overlapping circles of diameter $D$ whose centers are displaced a distance $\rho$ :

$$
\begin{aligned}
K_{D}(\rho) & =\frac{4}{\pi D^{2}} \int \mathrm{d} \boldsymbol{R} W\left(\boldsymbol{R}+\frac{1}{2} \rho\right) W\left(\boldsymbol{R}-\frac{1}{2} \rho\right) \\
& =\frac{2}{\pi}\left\{a \cos \left(\frac{\rho}{D}\right)-\left(\frac{\rho}{D}\right)\left[1-\left(\frac{\rho}{D}\right)^{\frac{1}{2}}\right]\right\}, \quad \rho \leq D .
\end{aligned}
$$

Note that the single integral in Eq. (4) can be characterized as a coherent effective area $A_{\text {eff }}$ of field fluctuations, in which case the value of $\Omega$,

$$
\Omega=\frac{4}{\pi D^{2}} A_{\text {eff }}
$$

decreases with the ratio of the receiver measurement area to $A_{\text {eff }}$. Note that the fading amplitude meansquare value $\Omega$ corresponds to the so-called aperture efficiency, a parameter describing the effective portion of the aperture area $\pi D^{2} / 4$ collecting heterodyne optical power [22]. Thus the inverse $1 / \Omega$ can be interpreted as the average number of field coherent areas (spatial modes of the field pattern) influencing the measurement area. Consistent with this interpretation of $1 / \Omega$, the average number of photons detected on the receiver aperture from a single pulse, i.e., the average SNR per pulse $\bar{\gamma}=\gamma_{0} \overline{a^{2}}=\gamma_{0} \Omega$, is the effective photocount coherently detected from a single pulse in a correlation area of the field pattern on the aperture plane. The exact number of spatial modes $1 / \Omega$ in the field pattern affecting the measurement area depends on the field spatial coherence and the area of integration of the aperture. Exact expressions for $\Omega$ can only be found when the correlation function $\mu(\rho)$ is specified. That being said, in the case of a receiver area that is very large compared with the average correlation length of the field fluctuations, the receiver aperture function $K_{D}(\rho)$ is much wider than the autocorrelation function $\mu(\rho)$, and we can factor out $K_{D}(\rho=0)=1$ from the integral in Eq. (田):

$$
\Omega \approx \frac{4}{\pi D^{2}} \int \mathrm{d} \rho \mu(\rho)
$$

where $\rho \leq D$. On the other hand, when the field correlation area is much wider than the aperture area, in Eq. (4) we can simplify $\mu(\rho)=1$ and, using the integral of $K_{D}$ equal to the aperture area $\pi D^{2} / 4$, the result is

$$
\Omega \approx \frac{4}{\pi D^{2}} \int \mathrm{d} \rho K_{D}(\rho)=1 .
$$

As we would have expected, in this limit, the number of field coherent areas affecting the measurement area approaches unity.

\section{A. Coherent Detection in Atmospheric Turbulence}

The presence of atmospheric turbulence needs to be considered into the statistical description of coherent lidar return signals. Turning attention to fading $\boldsymbol{\alpha}$, we study how amplitude and phase turbulenceinduced fluctuations of the optical field define the 
statistics of the fading intensity $\alpha^{2}=\alpha_{r}^{2}+\alpha_{i}^{2}$. We note that the two random magnitudes $\alpha_{r}$ and $\alpha_{i}$ are expressed in Eq. (11) as integrals over the aperture and, hence, are the sums of contributions from each point in the aperture. In order to proceed with the analysis, we could consider a statistical model in which these continuous integrals are expressed as finite sums over $N$ statistically independent cells in the aperture. Under the assumption that the number of independent coherent regions $N$ is large enough, we can consider that $\alpha_{r}$ and $\alpha_{i}$ asymptotically approach jointly normal random variables. Then, the probability density function of the length fading amplitude $\alpha$ can be well approximated by a Rayleigh distribution.

Just as in a speckle pattern, the Rayleigh distribution for the turbulence amplitude fading length is a consequence of the central-limit theorem. However, under conditions of weak turbulence in which the number of coherent terms is small, the fading may actually be the result of summing a small number of terms. In this case, the fading $\alpha$ is not likely to be Rayleigh. Rather than assuming that $\alpha$ is always Rayleigh distributed for all conditions of turbulence, it is more realistic to assume that $\alpha$ satisfied a generalized Rayleigh distribution that becomes Rayleigh only in the limit as the number of coherent terms $N$ becomes large. Such a distribution is the Nakagami-m distribution [23], which in essence is a central chi-square distribution described by

$$
p_{\alpha}(\alpha)=2(m N)^{m} \frac{\alpha^{2 m-1}}{\Gamma(m)} \exp \left(-m N \alpha^{2}\right)
$$

Here, $\Gamma$ is the complete gamma function. The parameter $m$ characterizes the amount of turbulent fading. When $m \rightarrow 1$, the number of contribution coherent areas $N$ is very large and the $m$ distribution reduces to Rayleigh. Note that the Nakagami-m distribution closely approximates the Rice distribution [23], which we previously used to model the impact of atmospheric turbulence-induced fading on freespace optical communication links using coherent detection [24]. Applying the Jacobian of the transformation $\alpha^{2}=\gamma / \gamma_{0}$, the corresponding SNR $\gamma$ distribution can be described according to a gamma distribution given by

$$
p_{\gamma}(\gamma)=\left(\frac{m N}{\gamma_{0}}\right)^{m} \frac{\gamma^{m-1}}{\Gamma(m)} \exp \left(-\frac{m N}{\gamma_{0}} \gamma\right) .
$$

When $m \rightarrow 1$, the gamma distribution reduces to exponential distribution. The Nakagami-m parameter $m$ and fading parameter $N$ are measures of turbulence effects. Here, $N$ is the inverse of the fading mean-square value

$$
N=1 / \Omega=1 / \overline{a^{2}}
$$

and, according to Eq. (6), it describes the number of field coherent areas affecting the fading measurement. Also, it can be shown that the distribution moments are given by

$$
\bar{\gamma}^{k}=\frac{\Gamma(m+k)}{m^{k} \Gamma(m)} \bar{\gamma}^{k},
$$

which yields, by using the average SNR $\bar{\gamma}=\gamma_{0} \Omega=$ $\gamma_{0} \overline{a^{2}}$, an expression for $m$

$$
\frac{1}{m}=\frac{\sigma_{\gamma}^{2}}{\bar{\gamma}^{2}}=\frac{\overline{a^{4}}}{\overline{a^{2}}}-1 .
$$

Since $\alpha_{r}$ and $\alpha_{i}$ can be considered jointly normal random variables, it is possible to relate high-order moments with the lower-order moments and replaces the fourth-order moment in Eq. (13) by $\overline{a^{4}}=$ $3{\overline{a^{2}}}^{2}-2 \overline{a^{4}}$. It results in

$$
\frac{1}{m}=2-2\left(\frac{\overline{a^{2}}}{\overline{a^{2}}}\right)^{2} .
$$

Although a Nakagami-m distribution can be reduced to a Rayleigh distribution, the parameter $m$, by characterizing the amount of fading through the normalized SNR $\gamma$ variance, gives more control over the extent of the turbulence fading. When $m \rightarrow 1$ and the number of contribution coherent areas is large, the normalized variance is one, as expected for Rayleigh distributions. When $m$ grows $m \rightarrow \infty$, and a very small number of contribution terms combine, the normalized variance decreases. Here, the density function becomes highly peaked around the mean value $\bar{\gamma}=\gamma_{0} / N$ and there is only a small fading to be considered.

To produce a measure of turbulence effects, it would be necessary to develop procedures to estimate $m$ and $N$. Equivalently, as Eqs. (11) and (14) describe fading parameters $m$ and $N$ in terms of fading first and second moments, we need to establish closed expressions for $\bar{\alpha}$ and $\overline{a^{2}}$. In order to assess the impact of turbulence, both log-amplitude and phase fluctuations should be considered. As a consequence, the complex field fluctuation pattern in the pupil plane is expressed as

$$
E_{S}(\boldsymbol{r})=\exp [\chi(\boldsymbol{r})-j \phi(\boldsymbol{r})]
$$

where $\chi(\boldsymbol{r})$ and $\phi(\boldsymbol{r})$ represent the log-amplitude fluctuations (scintillation) and phase variations (aberrations), respectively, introduced by atmospheric turbulence. Bringing the averaging operator into Eq. (11), we find the mean fading amplitude

$$
\begin{aligned}
\bar{\alpha} & =\frac{4}{\pi D^{2}} \overline{\int \mathrm{d} \boldsymbol{r} W(\boldsymbol{r}) E_{S}(\boldsymbol{r})} \\
& =\frac{4}{\pi D^{2}} \int \mathrm{d} \boldsymbol{r} W(\boldsymbol{r}) \overline{\exp [\chi(\boldsymbol{r})-j \phi(\boldsymbol{r})]} .
\end{aligned}
$$


The mean log amplitude can be extracted out of the integral, and assuming independence of $\chi$ and $\phi$, yields

$$
\bar{\alpha}=\frac{4}{\pi D^{2}} \exp (\bar{\chi}) \int \mathrm{d} \boldsymbol{r} W(\boldsymbol{r}) \overline{\exp [\chi(\boldsymbol{r})-\bar{\chi}] \exp [-j \phi(\boldsymbol{r})]} .
$$

We note that, because $\alpha$ results from atmospheric turbulence, we can consider phases $\phi$ that obey homogeneous, isotropic, zero-mean Gaussian statistics. Subject to this assumption, and the expressions for the mean of exponential functions of Gaussian variables, we can write

$$
\overline{\exp (-j \phi)}=\exp \left(-\frac{1}{2} \sigma_{\phi}^{2}\right)
$$

The phase variance $\sigma_{\phi}^{2}$ frequently is used to characterize the statistics of phase aberrations caused by atmospheric turbulence in considering a Kolmogorov spectrum of turbulence [25]:

$$
\sigma_{\phi}^{2}=1.0299\left(\frac{D}{r_{0}}\right)^{5 / 3}
$$

The coefficient 1.0299 in the phase variance $\sigma_{\phi}^{2}$ assumes that no terms are corrected by a receiver employing active modal phase compensation. In Eq. (19), the receiver aperture diameter $D$ is normalized by the wavefront coherence diameter $r_{0}$, which describes the spatial correlation of phase fluctuations in the receiver plane [12]. If it is also assumed that the log amplitudes $\chi$ are normal random variables [26], we can use energy conservation, and the mean of exponential functions of normal variables, to obtain classical results for the log-amplitude and amplitude means:

$$
\bar{\chi}=-\sigma_{\chi}^{2}, \quad \overline{\exp (\chi-\bar{\chi})}=\exp \left(\frac{1}{2} \sigma_{\chi}^{2}\right) .
$$

The mean of the irradiance $\beta \equiv \exp 2\left(\chi_{k}-\bar{\chi}\right)$ is given by $\exp \left(2 \sigma_{\chi}^{2}\right)$. The log-amplitude variance $\sigma_{\chi}^{2}$ is often expressed as a scintillation index $\sigma_{\beta}^{2}=\exp \left(4 \sigma_{\chi}^{2}\right)-1$. Replacing Eqs. (18) and (20) into Eq. (17), and noting that the integral of the aperture function $W(r)$ is simply the aperture area $\pi D^{2} / 4$, yields

$$
\bar{\alpha}=\exp \left(-\frac{1}{2} \sigma_{\chi}^{2}\right) \exp \left(-\frac{1}{2} \sigma_{\phi}^{2}\right) .
$$

Equation (4) describes the fading mean-square value $\overline{a^{2}}$ in terms of the field fluctuation coherence function $\mu(\rho)$. In atmospheric turbulence, based on the fact that the statistics of phase and log amplitude are homogeneous, isotropic, and Gaussian, we have [12]

$$
\begin{aligned}
\mu(\boldsymbol{\rho}) & =\overline{\exp \left\{\left[\chi(\boldsymbol{r})+\chi\left(\boldsymbol{r}^{\prime}\right)\right]-j\left[\phi(\boldsymbol{r})-\phi\left(\boldsymbol{r}^{\prime}\right)\right]\right\}} \\
& =\exp \left[-\frac{1}{2} D_{W}(\rho)\right]
\end{aligned}
$$

where $\rho=\left|\boldsymbol{r}-\boldsymbol{r}^{\prime}\right|$. Here, based on the Kolmogorov theory of turbulence, the wave structure function $D_{W}(\rho)$ describes the statistics of optical field variation in the atmosphere in terms of the coherence diameter $r_{0}$ as

$$
D_{W}(\rho)=6.88\left(\frac{\rho}{r_{0}}\right)^{\frac{5}{3}} .
$$

By replacing Eqs. (22) and (23) into Eq. (4), we obtain an explicit expression for the fading $\overline{a^{2}}$

$$
\overline{a^{2}}=\frac{4}{\pi D^{2}} 2 \pi \int_{0}^{D / 2} \rho \mathrm{d} \rho K_{D}(\rho) \exp \left[-\frac{1}{2} 6.88\left(\frac{\rho}{r_{0}}\right)^{\frac{5}{3}}\right],
$$

where we note that the integrand is an isotropic function of $\rho$, so that the angular part of the $\rho$ integration in Eq. (四) can be performed with minimal effort. In the case of a receiver area that is large compared with the average correlation length of the field fluctuations, we can factor out $K_{D}(\rho=0)=1$ from the integral and solve it in a closed form to obtain

$$
\overline{a^{2}}=1.09\left(\frac{r_{0}}{D}\right)^{2} \Gamma\left[\frac{6}{5}, 1.08\left(\frac{D}{r_{0}}\right)^{5 / 3}\right] .
$$

Here, $\Gamma(a, x)$ is the lower incomplete gamma function. Physical insight into this result may be obtained by considering the limiting case in which the receiver aperture is much greater than the coherence diameter $r_{0}$, i.e., $D \gg r_{0}$. In this case, the lower incomplete gamma function in Eq. (25) can be replaced by the gamma function $\Gamma(6 / 5)$ to obtain $\overline{a^{2}}=$ $1.007\left(r_{0} / D\right)^{2}$. To a good approximation, being the inverse of the fading mean-square value of the number of field coherent areas affecting the fading measurement $N$ [Eq. (11)], the aperture can be considered to consist of $\left(D / r_{0}\right)^{2}$ independent cells, each of diameter $r_{0}$.

By using Eqs. (21) and (24) in Eqs. (11) and (14), it is simple to express the proposed Nakagami-m parameters $N$ and $m$ in terms of three well-known magnitudes in atmospheric turbulence studies: the log-amplitude variance $\sigma_{\chi}^{2}$, often used to characterize the statistics of amplitude fluctuations; the phase variance $\sigma_{\phi}^{2}$, needed to depict the statistics of phase aberrations; and the wave structure function $D_{W}(\rho)$ which, depending on the aperture diameter $D$ normalized by the wavefront coherence diameter $r_{0}$, describes the spatial correlation of phase fluctuations in the receiver plane.

\section{B. Speckle Coherent Detection Statistics}

One principal performance limitation encountered by lidar receivers is produced by target speckle 
processes that appear because the backscattered signal is composed of a multitude of independent phased additive complex components. When only target speckle is considered, Eq. (11) represents the integral of the complex field of a speckle pattern that, by the Central Limit Theorem-the number of random phasors contributing from a rough target to the speckle pattern is large-it is a circular complex Gaussian random process over space. The integral represents a linear transformation of that process and, consequently, must also obey circular complex Gaussian statistics [27]. It follows that the channel fading amplitude $\alpha$ must obey Rayleigh statistics

$$
p_{\alpha}(\alpha)=2 M \alpha \exp \left(-M \alpha^{2}\right),
$$

where the inverse of the fading mean-square value $M=1 / \Omega$ represents, consistent with the previous interpretation, the average number of speckles influencing the coherent measurement. Hence, using the average SNR $\bar{\gamma}=\gamma_{0} \Omega=\gamma_{0} / M$ and the Jacobian of the transformation $\alpha^{2}=\gamma / \gamma_{0}$, the instantaneous SNR per pulse $\gamma$ is distributed according to an exponential distribution given by

$$
p_{\gamma}(\gamma)=\frac{M}{\gamma_{0}} \exp \left(-\frac{M}{\gamma_{0}} \gamma\right)
$$

In a more general case of speckle, where the return signal is defined not by a single speckle pattern but rather by the sum of $n$ independent speckle patterns generated when $n$ identical laser shots are averaged, the exponential distribution in Eq. (27) becomes a gamma density function of order $n$ [27]:

$$
p_{\gamma}(\gamma)=\left(\frac{n M}{\gamma_{0}}\right)^{n} \frac{\gamma^{n-1}}{\Gamma(n)} \exp \left(-n M \frac{\gamma}{\gamma_{0}}\right)
$$

The parameter $M=1 / \Omega$ determines the statistics of the speckled SNR $\gamma$ and, consequently, we need to consider it closely. The number of spatial speckle modes $M$ depends on the correlation function of speckle $\mu_{S}$ and the receiving aperture function $K_{D}$. In the case of a Gaussian-shaped field pattern on the scattering target, and by virtue of the van Cittert-Zernike theorem described by the Fourier integral in the spatial coherence function $\mu_{S}$ that propagates from the spatially incoherent target plane $\nu$, we get

$$
\mu_{S}(\rho)=\frac{2}{\pi W_{T}^{2}} \int \mathrm{d} \nu \exp \left(-\frac{2 \rho^{2}}{W_{T}^{2}}\right) \exp \left(-j \frac{k}{z} \nu \cdot \rho\right) .
$$

After solving the Fourier integral, the result is

$$
\mu_{S}(\rho)=\exp \left[-\frac{1}{2}\left(\frac{k W_{T}}{2 z} \rho\right)^{2}\right] \equiv \exp \left[-\left(\frac{\rho}{\rho_{S}}\right)^{2}\right] .
$$

Here, $\rho_{S}$ is defined as the speckle coherence radius describing the spatial size of the field correlated areas

$$
\rho_{S}=\sqrt{2}\left(\frac{2 z}{k W_{T}}\right),
$$

where $k=2 \pi / \lambda$ is the free-space wavenumber and $z$ is the distance to the target. $W_{T}$ is a measure of the transmitted laser beam width at the target plane. In atmospheric propagation, where the presence of refractive turbulence must be taken into account, the intensity $1 / e^{2}$ radius $W_{T}(z)$ for an initially collimated beam can be approximated well as [28]

$$
W_{T}^{2}(z)=W_{0}^{2}\left[1+\left(\frac{z}{z_{0}}\right)^{2}\right]+2\left(\frac{4 z}{k r_{0}}\right)^{2} .
$$

The first term in Eq. (32) is the free-space beam intensity radius, $W_{0}$ is the initial $1 / e^{2}$ intensity radius, and $z_{0}=k W_{0}^{2} / 2$ is the Rayleigh range of the beam. The term $4 z / k r_{0}$ in Eq. (32) describes the additional beam spreading introduced by turbulence. The wavefront coherence diameter $r_{0}$ describes turbulence for the path where the point source is located at the scattering target plane and evaluated at the lidar location (27). It is important to note that the expressions for $\mu_{S}(\rho)$ given by Eqs. (29) and (31) only consider atmospheric turbulence on the one-way path from the lidar to the target scattering region of interest. Because, in lidar applications, we are concerned with a round-trip path, we also need to introduce turbulence effects on the return path from the target plane to the lidar location. If we assume that atmospheric turbulence is statistically independent of the fluctuations associated with the rough target, the field degree of coherence in Eq. (29) must be modified as

$$
\mu_{S}(\rho)=\exp \left[-\frac{1}{2}\left(\frac{k W_{T}}{2 z} \rho\right)^{2}\right] \exp \left[-\frac{1}{2} 6.88\left(\frac{\rho}{r_{0}}\right)^{\frac{5}{3}}\right] .
$$

Based on the Kolmogorov theory of turbulence, we used Eqs. (22) and (23) to describe the loss of field coherence due to phase and log-amplitude fluctuations in atmospheric turbulence. Equation (33) is equivalent to Eq. (30) by rewriting the exponential arguments in terms of a modified speckle coherent radius. By noting that the argument of the turbulence exponential in Eq. (33) can be approximated as $\left(2 \rho / r_{0}\right)^{5 / 3}$, and replacing $5 / 3$ by 2 in the exponent, we can obtain a good analytical expression for the speckle coherence radius affected by round-trip turbulence:

$$
\frac{1}{\rho_{S}^{2}}=\frac{1}{2}\left(\frac{k W_{T}}{2 z}\right)^{2}+\frac{4}{r_{0}^{2}}
$$

Here, the coherence diameter $r_{0}$ in the receiver plane refers to the round-trip atmospheric path and is a factor of $4^{-3 / 5}$ less than the corresponding coherence length for the one-way path used in Eq. (32) [13]. In the limit of weak atmospheric turbulence, the degree of coherence of the backscattered field is not strongly 
affected by atmospheric turbulence, the term $4 / r_{0}^{2}$ can be excluded, and Eq. (34) becomes the one-way path speckle coherence radius in Eq. (31). Also, in this limit, the turbulence spreading in Eq. (32) can be excluded and the beam radius can be approached by $W_{T} \approx 2 z / k W_{0}$. In this case of weak turbulence, Eq. (31) leads to the result $\rho_{S} \approx \sqrt{2} W_{0}$. On the other hand, in the limit of strong atmospheric turbulence, the first term in Eq. (34) can be excluded as turbulence dominates the speckle coherence, and we can approach $2 \rho_{S} \approx r_{0}$.

In the case of a circular aperture, by replacing the speckle correlation function $\mu_{S}$ in Eq. (29) into Eq. (4), we obtain an expression for the parameter $\Omega=1 / M$ describing the average number of speckle areas in the receiving aperture $D$ :

$$
\Omega=\frac{4}{\pi D^{2}} \int \mathrm{d} \rho K_{D}(\rho) \exp \left[-\left(\frac{\rho}{\rho_{S}}\right)^{2}\right] .
$$

Here, the speckle coherence diameter $\rho_{S}$ is given by Eq. (34). By assuming a receiving area that is large compared with the speckle diameter $2 \rho_{S}$, we can factor out $K_{D}(\rho=0)=1$ from the integral, to obtain the closed-form expression

$$
\begin{aligned}
\frac{1}{M} & =\frac{4}{\pi D^{2}} 2 \pi \int_{0}^{D / 2} \rho \mathrm{d} \rho \exp \left[-\left(\frac{\rho}{\rho_{S}}\right)^{2}\right] \\
& =\left(\frac{2 \rho_{S}}{D}\right)^{2}\left\{1-\exp \left[-\left(\frac{D}{2 \rho_{S}}\right)^{2}\right]\right\} .
\end{aligned}
$$

Physical insight is obtained by considering the limiting case where $D \gg 2 \rho_{S}$. To a good approximation, the aperture can be considered to consist of $M=$ $\left(D / 2 \rho_{S}\right)^{2}$ independent cells, each of diameter $2 \rho_{S}$.

\section{Detection Statistics in Heterodyne Lidars}

Detection of rough targets in a turbulent atmosphere requires that both the fluctuations of the target and the fluctuations of turbulence be taken into account (see Fig. 11). For typical atmospheric situations, the time scale of the fluctuations due to turbulence is several orders of magnitude larger than that of speckle-induced fluctuations (milliseconds rather than microseconds). The long time constant of the return signal fluctuation due to turbulence means that these fluctuations are essentially correlated over the short correlation time associated with speckle. Consequently, the mean of the target speckle signal is smeared by turbulence speckle fluctuations. There must be a compounding of the statistics for the signal affected by speckle Rayleigh fading, conditioned on knowledge of the mean value as described by the Nakagami-m distribution characterizing the signal affected by turbulence. Here, the speckle process is driven by the turbulence random process, and the problem must be analyzed by the application of conditional speckle statistics.

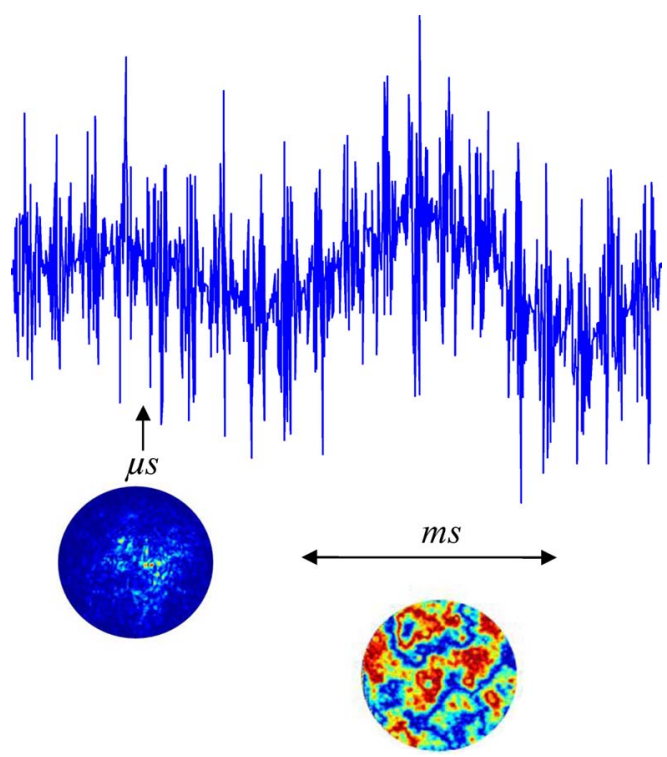

Fig. 1. (Color online) For typical atmospheric situations, the time scale of the coherent lidar signal fluctuations due to turbulence is several orders of magnitude larger than that of speckle-induced fluctuations (milliseconds rather than microseconds). The long time constant of the return signal fluctuation due to turbulence means that these fluctuations are essentially correlated over the short correlation time associated with speckle. Here, the mean of the target speckle signal is smeared by turbulence speckle fluctuations, and we need to define multiply stochastic (compound) statistics to describe the return signals in a coherent lidar.

We can regard the speckle distribution Eq. (28) to be a conditional density function, conditioned on knowledge of the SNR $\gamma_{0}$, which we represent here by the variable $x$ :

$$
p_{\gamma \mid x}(\gamma \mid x)=\left(\frac{n M}{x}\right)^{N} \frac{\gamma^{n-1}}{\Gamma(n)} \exp \left(-n M \frac{\gamma}{x}\right) .
$$

The unconditional probability density function of the SNR $\gamma$ is found by averaging the above density function with respect to the statistics of the conditional SNR $x$ :

$$
\begin{aligned}
p_{\gamma}(\gamma)= & \int_{0}^{\infty} p_{\gamma \mid x}(\gamma \mid x) p_{x}(x) \mathrm{d} x=(n M)^{n} \frac{\gamma^{n-1}}{\Gamma(n)} \int_{0}^{\infty}\left(\frac{1}{x}\right)^{n} \\
& \times \exp \left(-n M \frac{\gamma}{x}\right) p_{x}(x) \mathrm{d} x .
\end{aligned}
$$

By assuming that the speckle is driven by turbulence, we indicate that $p_{x}(x)$ ) obeys the gamma distribution in Eq. (10):

$$
p_{x}(x)=\left(\frac{m N}{\gamma_{0}}\right)^{m} \frac{x^{m-1}}{\Gamma(m)} \exp \left(-\frac{m N}{\gamma_{0}} x\right)
$$

and, after some simple algebra, the result of the integration in Eq. (37) can be reduced to 
$p_{\gamma}(\gamma)=\frac{2}{\Gamma(n) \Gamma(m)}\left(\frac{n M m N}{\gamma_{0}} \gamma\right)^{\frac{n+m}{2}} \frac{1}{\gamma} K_{m-n}\left[2\left(\frac{n M m N}{\gamma_{0}} \gamma\right)^{\frac{1}{2}}\right]$.

Here, $K_{\beta}$ is a modified Bessel function of the second kind, order $\beta$. This result is a generalization of the well-known $K$ distribution. Equation (40) is the main result of this modeling effort. The model results in a three-parameter probability distribution for the coherent SNR in the presence of atmospheric turbulence and affected by target speckle. These parameters are the average number of speckles influencing the coherent measurement $M$, the number of turbulent coherent areas in the receiver affecting the fading measurement $N$, and the amount of fading introduced by atmospheric turbulence in the lidar signal $1 / m$. These three parameters, along with the number of averaged shots $n$ and the turbulence-free photocount budget $\gamma_{0}$, completely characterize the statistics of return fading signals in coherent lidars.

\section{Performance Analysis of Coherent Lidars}

The most common and well-understood performance measures of a coherent lidar system in the presence of fading are the average SNR $\bar{\gamma}$ and the normalized variance $\sigma_{\gamma}^{2} / \bar{\gamma}^{2}$. They describe the inherent statistical uncertainty of the process of heterodyne SNR $\gamma$ measurement because of the presence of refractive turbulence and speckle. In order to describe these two performance measures, we need to know at least the first statistical moments of the instantaneous SNR $\gamma$. As a function of the independent laser shots $n$ averaged at the receiver, with some algebra, the moments about the origin of the atmospheric $\mathrm{SNR} \gamma$ can be calculated from Eq. (40) in terms of the turbulence-free SNR $\gamma_{0}$ (detected photocounts), the fading parameter $m$ characterizing the strength of atmospheric turbulence, and the number of spatial modes $M$ and $N$ that describe target speckle and atmospheric turbulence, respectively,

$$
\bar{\gamma}^{k}=\int_{0}^{\infty} \mathrm{d} \gamma \gamma^{k} p_{\gamma}(\gamma)=\frac{\Gamma(k+m)}{\Gamma(m)} \frac{\Gamma(k+n)}{\Gamma(n)}\left(\frac{\gamma_{0}}{n M m N}\right)^{k} .
$$

From here, the mean SNR can be written as

$$
\bar{\gamma}=\frac{\gamma_{0}}{M N},
$$

indicating that the strength of the detected signal is determined by the number of photons falling on the turbulence effective area per speckle correlation area. Of special interest is the ratio of the SNR variance to its mean square that describes the uncertainty in the lidar measurement process:

$$
\frac{\sigma_{\gamma}^{2}}{\bar{\gamma}^{2}}=\frac{\Gamma(m) \Gamma(m+2)}{\Gamma^{2}(m+1)} \frac{\Gamma(n) \Gamma(n+2)}{\Gamma^{2}(n+1)}-1 .
$$

With $m$ being a positive real number, Eq. (43) simplifies to

$$
\frac{\sigma_{\gamma}^{2}}{\bar{\gamma}^{2}}=\frac{(m+1)}{m} \frac{(n+1)}{n}-1 .
$$

Note that, because the heterodyne lidar SNR results from compounding two sources of fluctuation (atmospheric turbulence and target speckle), this magnitude could be greater than unity.

\section{A. Noncompensated Passive Receivers}

Figure 2 shows the effect of atmospheric turbulence and speckle on the performance of Doppler lidars for passive receivers, those nonemploying active modal compensation of wavefront phase distortion. We study the mean SNR $\bar{\gamma}$ in Fig. 2(a) and the SNR normalized variance $\sigma_{\gamma}^{2} / \bar{\gamma}^{2}$ in Fig. 2(b) as functions of several parameters: the average turbulence-free SNR $\gamma_{0}$, the receiver aperture diameter $D$, the number of speckle modes $M$, and the strength of atmospheric turbulence. Turbulence is quantified by two parameters: the phase coherence length $r_{0}$ and the scintillation index $\sigma_{\beta}^{2}$. The value of the scintillation index $\sigma_{\beta}^{2}=1$ corresponds to strong scintillation, but still below the saturation regime. When we assume no scintillation, $\sigma_{\beta}^{2}=0$, the effect of turbulence is simply to reduce the coherence length $r_{0}$. For a fixed coherent diameter $r_{0}$, as aperture diameter $D$ is increased, the normalized aperture diameter $D / r_{0}$ increases, and turbulence reduces the heterodyne downconversion efficiency.

In Fig. 2(a), the mean SNR $\bar{\gamma}=\gamma_{0} / M N$ is plotted against the normalized aperture diameter $D / r_{0}$ for different values of the speckle coherent diameter $2 \rho_{S}$. The SNR is expressed in decibels, referenced to the smallest aperture considered in the figure. The speckle coherence diameter $2 \rho_{S}$ is expressed in terms of the turbulence coherence diameter $r_{0}$. We also consider the nonspeckle case by plotting $\bar{\gamma}=\gamma_{0} / N$ in Fig. 2(a). The mean SNR depends on the number of spatial modes $M$ and $N$ describing target speckle and atmospheric turbulence, but it is independent of both the amount of turbulence fading $m$ and the number of averaged shots $n$ accumulated. The effects of scintillation on the mean SNR $\bar{\gamma}$ are very weak and they have not been considered in Fig. 2(a). The noturbulence, no-speckle SNR $\rho_{0}$ is also presented in Fig. 2(a). For any aperture diameter, the value of $\gamma_{0}$ is proportional to $D^{2}$. As is expected when speckle is not considered [1], if $D$ is less than $r_{0}$, the mean SNR $\bar{\gamma}$ increases as the square of the diameter. When diameter $D$ is larger than $r_{0}$, atmospheric turbulence limits the effective receiving aperture to the dimensions of the coherence diameter $r_{0}$. This effective aperture defines the maximum possible coherent mean SNR $\bar{\gamma}$. When target speckle is taken into 
(a)

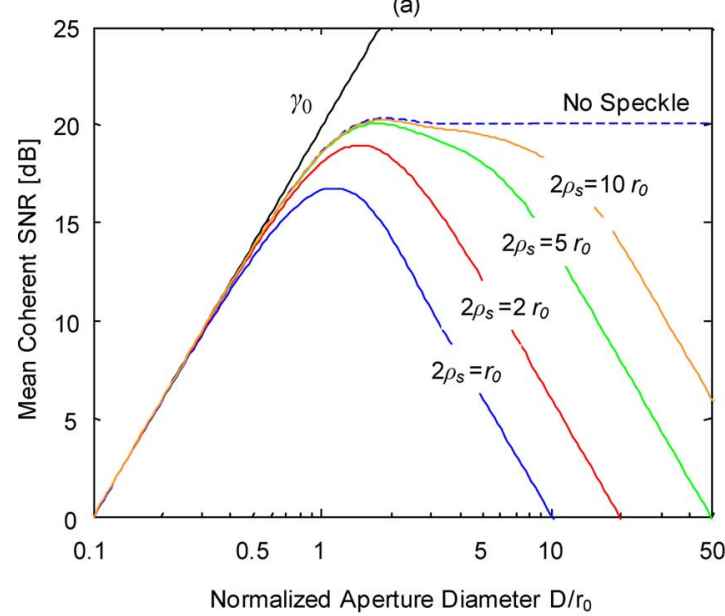

(b)

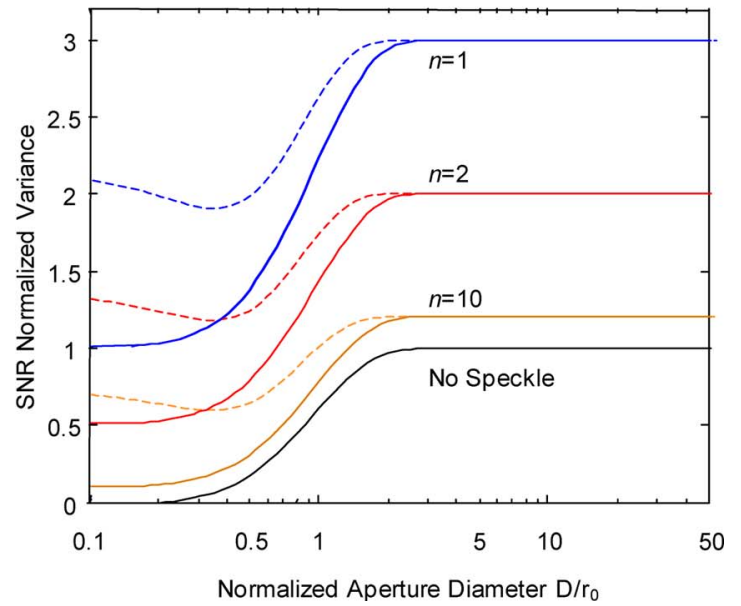

Fig. 2. (Color online) Mean coherent SNR $\bar{\gamma}$ (photocounts) and SNR normalized variance $\sigma_{\gamma}^{2} / \bar{\gamma}^{2}$ as a function of the normalized receiver aperture diameter $D / r_{0}$. (a) Mean coherent SNR is shown for different coherence diameters of spatial speckle $2 \rho_{S}$ over the receiving aperture. The SNR is expressed in decibels, referenced to the smallest aperture considered in the figure. The no-speckle case (dashed curve) and free-space limit $\gamma_{0}$ are included. (b) SNR normalized variance is studied when $n$ equal-strength independent laser shots are averaged. Amplitude fluctuations are excluded (solid curves) by assuming $\sigma_{\beta}^{2}=0$. When scintillation is considered (dashed curves), the scintillation index is fixed at $\sigma_{\beta}^{2}=1$.

account, the behavior of the mean SNR $\bar{\gamma}$ is markedly different. Here, for a fixed coherence diameter $r_{0}$, when the normalized aperture diameter $D / r_{0}$ is large, we increase the number of speckle coherent areas $M$ affecting the collected SNR. As a consequence, for large apertures the mean SNR $\bar{\gamma}$ goes down very quickly. For instance, when a large normalized aperture $D / r_{0}=10$ is considered, decreasing the speckle coherence diameter $2 \rho_{S}$ from $5 r_{0}$ to $10 r_{0}$ penalizes the mean SNR by more than $15 \mathrm{~dB}$.

In Fig. 2(b), we plot the normalized SNR variance $\sigma_{\gamma}^{2} / \bar{\gamma}^{2}$ against the normalized aperture diameter $D / r_{0}$ for different values of the number of averaged shots $n$ used to define the lidar signal. Each shot produces $\gamma_{0} / n$ undisturbed photons, so that the total received photocount in both the single and the multiple shots situation is the same $\gamma_{0}$. As shown by Eqs. (43) and (44), the magnitude $\sigma_{\gamma}^{2} / \bar{\gamma}^{2}$ depends on the number of averaged shots $n$ and the amount of turbulence fading $m$, but it is independent of the number of spatial modes $M$ and $N$ describing target speckle and atmospheric turbulence, respectively. In the limit of weak turbulence (small normalized aperture diameter $D / r_{0}$, i.e., $m \rightarrow \infty$ ), the normalized variance trends asymptotically to $1 / n$. For a single shot $n=1$, the normalized variance is unity, as expected for the classical negative exponential speckle. However, in the limit of strong turbulence (large normalized aperture diameter $D / r_{0}$, i.e., $m \rightarrow 1$ ), the normalized variance becomes $1+2 / n$. When $n=1$, we reach a maximum value of 3 . As we observe in Fig. 2(b), the effects of scintillation are noticeable for the small aperture diameters and must be properly considered. For relatively small apertures, amplitude scintillation is dominant, and normalized variance is virtually unaffected by wavefront phase distortions. When the aperture is larger, phase distortion becomes dominant and the normalized variance is substantially independent of the scintillation index $\sigma_{\beta}^{2}$.

\section{B. Active Modal-Compensated Receivers}

Adaptive compensation of atmospheric wavefront phase distortion to improve the performance of atmospheric systems is an important field of study. In particular, modal compensation method involves correction of several modes of an expansion of the total phase distortion in a set of basis functions. We can consider the effects of amplitude and phase fluctuations for receivers employing active modal compensation of wavefront phase distortion. Typically, only phase variations are compensated in adaptive systems, and we need to modify only the two magnitudes used to describe the statistics of wavefront phase fluctuations in atmospheric turbulence, i.e., the phase variance $\sigma_{\phi}^{2}$ and the wave structure function $D_{W}(\rho)$, according to the degree of compensation applied to the receiving system.

In [25], the statistics of phase aberrations caused by atmospheric turbulence were characterized considering a Kolmogorov spectrum of turbulence. In that analysis, classical results for the phase variance $\sigma_{\phi}^{2}$ were extended to consider modal compensation of atmospheric phase distortion. In such modal compensation, Zernike polynomials are widely used as basis functions because of their simple analytical expressions and their correspondence to classical aberrations [29]. It is known that the residual phase variance following modal compensation of $J$ Zernike terms is given by

$$
\sigma_{\phi}^{2}=C_{J}\left(\frac{D}{r_{0}}\right)^{5 / 3}
$$

where the aperture diameter $D$ is normalized by the wavefront coherence diameter $r_{0}$, which describes 
the spatial correlation of phase fluctuations in the receiver plane [12]. In Eq. (45), the coefficient $C_{J}$ depends on $J$ [25]. For example, aberrations up to tip-tilt, astigmatism, and coma correspond to $J=$ 3,6 , and 10 , respectively. These aberrations are on the scale of the largest wavefront distortions. Ideally, it is desirable to set $J$ large enough for the residual variance in Eq. (45) to become negligible. As the distribution on the size of the wavefront distortions that are due to turbulence approximately follows Kolmogorov's spectrum, this means that most of the turbulence power is on the previous largest scales aberrations. Removal of the variance due to these largest scales would increase the turbulence coherence diameter $r_{0}$ to a great extent. For example, compensating wavefront aberrations up to astigmatism $(J=6)$ reduces the residual phase variance by a factor of $15\left(C_{J}=0.0648\right)$, which is roughly equivalent to increasing the coherence diameter by a factor of 5 .

Completion of the statistical description of corrected wavefronts requires consideration of the spatial correlation of phase fluctuations. To consider the detection statistics of receivers employing active modal atmospheric compensation, a modified form of structure function $D_{W}(\rho)$ would be applied to the compensated wavefront. In a first approach, after modal correction of the first $J$ modes, we may be tempted to consider the standard structure function in Eq. (23) with, according to Eq. (45), a proportionally larger coherence diameter (as noted, aberration compensation up to astigmatism will produce a factor of 5 increase in coherence diameter). Certainly, a more accurate approach to the problem has been described [30]. For modal compensation, when the atmospheric wavefront phase is partially corrected, the residual structure function can be written analogously to the atmospheric phase structure function as [30]

$$
D_{W}(\rho)=6.44\left(\frac{\rho}{r_{0}}\right)^{\frac{5}{3}}-D_{J}(\rho) .
$$

The function $D_{J}(\rho)$, expressed in terms of the mode and cross-mode shape functions of Zernike polynomials [29] in this analysis, describes the compensation for the residual phase structure function after modal correction of the first $J$ modes.

Figure 3 shows the effect of atmospheric turbulence and speckle on the performance of Doppler lidars for receivers employing active modal compensation of wavefront phase distortion. We study the mean SNR $\bar{\gamma}$ in Fig. 3(a) and the normalized variance $\sigma_{\gamma}^{2} / \bar{\gamma}^{2}$ in Fig. 3(b) as a function of the receiver aperture diameter $D$, the number of speckle modes $M$, the strength of atmospheric turbulence, and the number of spatial modes $J$ removed by the compensation system. The compensating phases are expansions up to tilt-tilt $(J=3)$, astigmatism $(J=6)$, and fifth-order aberrations $(J=20)$. The no-turbulence case is also shown.

In Fig. $3(\mathrm{a})$, the mean SNR $\bar{\gamma}$ is considered. The speckle coherence diameter $2 \rho_{S}$ is fixed and equal

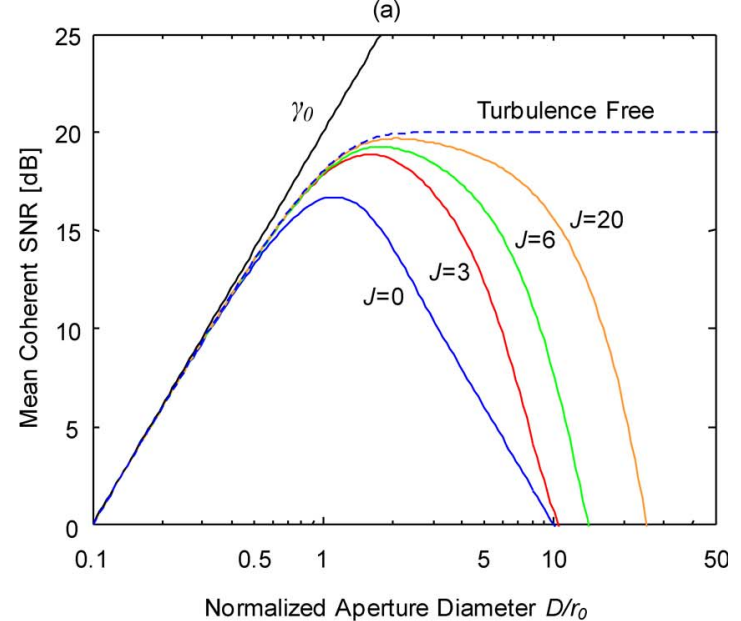

(b)

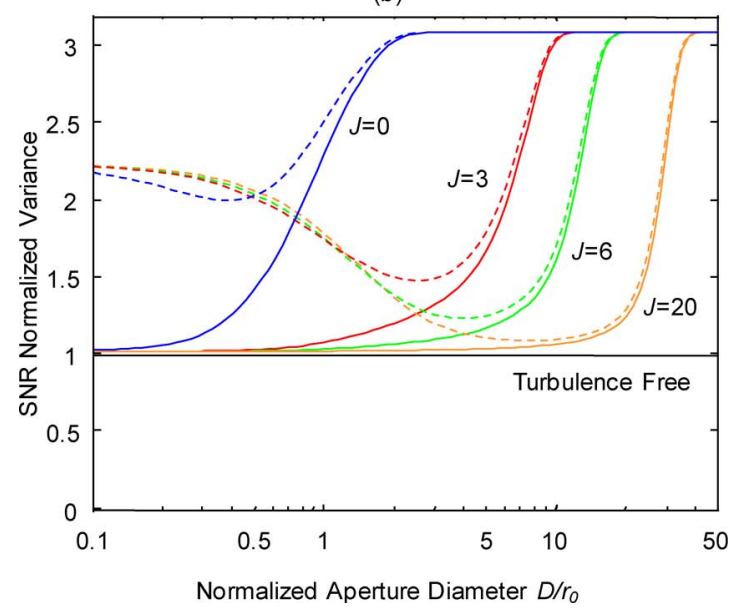

Fig. 3. (Color online) (a) Mean coherent SNR $\bar{\gamma}$ and (b) SNR normalized variance $\sigma_{\gamma}^{2} / \bar{\gamma}^{2}$ are shown for different values of the normalized receiver aperture diameter $D / r_{0}$ and the number of modes $J$ removed by phase compensation. Turbulence is characterized by a fixed phase coherence diameter $r_{0}$. Without loss of generality, in all cases considered in these plots, the correlation diameter of the speckle field $2 \rho_{S}$ is equal to $r_{0}$. The compensating phases are expansions up to tilt $(J=3)$, astigmatism $(J=6)$, and fifth-order aberrations $(J=20)$. The no-correction case $(J=0)$ is also considered. The dashed curve corresponds to the measurement associated with no-turbulence, one-pulse speckle. The free-space limit $\gamma_{0}$ is included in (a). (b) Amplitude fluctuations are excluded (solid curves) by assuming $\sigma_{\beta}^{2}=0$. When scintillation is considered (dashed curves), the scintillation index is fixed at $\sigma_{\beta}^{2}=1$.

to the coherence diameter $r_{0}$. No shot averaging is considered; i.e., $n=1$. We consider the nonturbulence case by plotting $\bar{\gamma}=\gamma_{0} / M$. We have found that for most typical lidar apertures, wavefront phase fluctuations are the dominant impairment, and compensation of a modest number of modes can reduce performance penalties by several decibels. For instance, when a large normalized aperture $D / r_{0}=$ 10 is considered, compensation of just $J=20$ modes increases the mean SNR $\bar{\gamma}$ by more than $15 \mathrm{~dB}$. Speckle effects are not diminished by the use of phase-compensation techniques.

In Fig. 3(b), we separately quantified the effects of amplitude fluctuations and wavefront phase 
distortion on the amount of turbulence fading $\sigma_{\gamma}^{2} / \bar{\gamma}^{2}$. The value of the scintillation index $\sigma_{\beta}^{2}=1$ is kept below the saturation regime. When the turbulence reaches the saturation regime, wavefront distortion becomes so severe that it would be unrealistic to consider phase compensation. We have identified two different regimes of turbulence based on the receiver aperture diameter that has been normalized to the coherence diameter of the wavefront phase. When the normalized aperture diameter is relatively small, amplitude scintillation dominates and, as phase fluctuations have little impact, performance is virtually independent of the number of modes compensated. For small normalized apertures, only when scintillation is excluded are the effects of phase compensation appreciable. For the single shot $n=1$ considered in Fig. 3(b), when no-turbulence is contemplated, the normalized variance is unity. When the normalized aperture is larger, amplitude fluctuations become negligible and phase fluctuations become dominant, so that high-order phase compensation may be needed to improve performance to acceptable levels. In any case, when no-compensation $J=0$ is used, the amount of fading introduced by turbulence is important even for apertures as small as the coherence diameter $r_{0}$. However, when $J=20$ modes are compensated, the amount of fading introduced by turbulence is a small fraction of speckle fading; thus it can be excluded even for apertures as large as ten times $r_{0}$.

\section{Conclusions}

We have focused on elucidating those implications of the atmospheric propagation problem that bear on the design and reliability of lidar coherent systems. We present recent studies on the impact of phase and amplitude fluctuations on Doppler lidars using coherent detection and consider, in a unified framework, the effects of wavefront distortion, amplitude scintillation, and diffuse target speckle on performance, for both passive receivers and those employing active modal compensation of wavefront phase distortion. As the effects ascribed to turbulence and speckle are random and must be quantified, we define a mathematical model for the probability density function of the received coherent signal after its propagation through the atmosphere. In our model, the parameters describing the signal statistics depend on turbulence and target conditions and the degree of modal compensation applied in the receiver. We provide analytical expressions for every key model parameter.

By noting that the impact of atmospheric turbulence on coherent receivers can be compounded with target speckle statistics, we have shown that the probability density function of the lidar return signal is an example from the family of $K$ distributions. Our formulation circumvents the need for a detailed description of the compound speckle and turbulence problem. Such a specification is difficult because of the inherent complexity of the propagation as well as the random distribution of the numerous target scatters. Our statistical model is developed from the fundamental principles of scattering and turbulence. This model results in a three-parameter distribution for the return signal, thereby making it possible to gain information on the physical properties of target and atmospheric turbulence underlying the parameter definitions. In this analysis, we estimate the parameters using a heuristic theory of coherent signals.

We have provided analytical expressions for the signal statistical moments and have used them to study the effect of various parameters on performance, including turbulence level, signal strength, receiver aperture size, speckle effective area, and the extent of compensation. We have separately quantified the effects of amplitude fluctuations and wavefront phase distortion on system performance and have identified different regimes of turbulence, depending on the receiver aperture diameter normalized to the coherence diameter of the wavefront phase. When the normalized aperture is larger, amplitude fluctuations become negligible, and phase fluctuations become dominant. We have found that, for most typical lidar situations, compensation of a modest number of modes can reduce performance penalties by several decibels.

This study was partially funded by the Spanish Ministry of Science and Innovation (MICINN) grant TEC 2009-10025.

\section{References and Notes}

1. J. Totems, V. Jolivet, J.-P. Ovarlez, and N. Martin, "Advanced signal processing methods for pulsed laser vibrometry," Appl. Opt. 49, 3967-3979 (2010).

2. D. Jameson, M. Dierking, and B. Duncan, "Effects of spatial modes on ladar vibration signature estimation," Appl. Opt. 46, 7365-7373 (2007).

3. M. J. Kavaya, "Laser and lidar technology development for highly accurate vertical profiles of vector wind velocity from earth orbit," in Coherent Optical Technologies and Applications (Optical Society of America, 2008), paper CTuA3.

4. S. Kameyama, T. Ando, K. Asaka, Y. Hirano, and S. Wadaka, "Compact all-fiber pulsed coherent Doppler lidar system for wind sensing," Appl. Opt. 46, 1953-1962 (2007).

5. A. Dinovitser, M. W. Hamilton, and R. A. Vincent, "Stabilized master laser system for differential absorption lidar," Appl Opt. 49, 3274-3281 (2010).

6. L. Joly, F. Marnas, F. Gibert, D. Bruneau, B. Grouiez, P. H. Flamant, G. Durry, N. Dumelie, B. Parvitte, and V. Zéninari, "Laser diode absorption spectroscopy for accurate $\mathrm{CO}_{2}$ line parameters at $2 \mu \mathrm{m}$ : consequences for space-based DIAL measurements and potential biases," Appl. Opt. 48, 5475-5483 (2009).

7. G. J. Koch, B. W. Barnes, M. Petros, J. Y. Beyon, F. Amzajerdian, J. Yu, R. E. Davis, S. Ismail, S. Vay, M. J. Kavaya, and U. N. Singh, "Coherent differential absorption lidar measurements of $\mathrm{CO}_{2}$," Appl. Opt. 43, 5092-5099 (2004).

8. S. Lundqvist, C.-O. Fält, U. Persson, B. Marthinsson, and S. T. Eng, "Air pollution monitoring with a $Q$-switched $\mathrm{CO}_{2}$-laser lidar using heterodyne detection," Appl. Opt. 20, 2534-2538 (1981).

9. Y. Zhao, W. A. Brewer, W. L. Eberhard, and R. J. Alvarez, "Lidar measurement of ammonia concentrations and fluxes 
in a plume from a point source," J. Atmos. Ocean. Technol. 19, 1928-1938 (2002).

10. A. Pal, C. D. Clark, M. Sigman, and D. K. Killinger, "Differential absorption lidar $\mathrm{CO}_{2}$ laser system for remote sensing of TATP related gases," Appl. Opt. 48, B145-B150 (2009).

11. See papers on Advanced Component Technologies presented at the Fifteenth Biennial Coherent Laser Radar Technology and Applications Conference (Universities Space Research Association, 2009).

12. D. L. Fried, "Optical heterodyne detection of an atmospherically distorted signal wave front," Proc. IEEE 55, 57-67 (1967).

13. H. T. Yura, "Signal-to-noise ratio of heterodyne lidar systems in the presence of atmospheric turbulence," Opt. Acta 26, 627-644 (1979).

14. J. H. Shapiro, B. A. Capron, and R. C. Harney, "Imaging and target detection with a heterodyne-reception optical radar," Appl. Opt. 20, 3292-3312 (1981).

15. S. F. Clifford and S. Wandzura, "Monostatic heterodyne lidar performance: the effect of the turbulent atmosphere," Appl. Opt. 20, 514-516 (1981).

16. S. F. Clifford and S. Wandzura, "Monostatic heterodyne lidar performance: the effect of the turbulent atmosphere; correction," Appl. Opt. 20, 1502 (1981).

17. B. J. Rye, "Refractive-turbulent contribution to incoherent backscatter heterodyne lidar returns," J. Opt. Soc. Am. 71, 687-691 (1981).

18. R. G. Frehlich and M. J. Kavaya, "Coherent laser radar performance for general atmospheric refractive turbulence," Appl. Opt. 30, 5325-5352 (1991).

19. J. L. Codona, D. B. Creamer, S. M. Flatté, R. G. Frehlich, and F. S. Henyey, "Solution for the fourth moment of waves propagating in random media," Radio Sci. 21, 929-948 (1986).

20. A. Belmonte and B. J. Rye, "Heterodyne lidar returns in turbulent atmosphere: performance evaluation of simulated systems," Appl. Opt. 39, 2401-2411 (2000).

21. A. Belmonte, "Influence of atmospheric phase compensation on optical heterodyne power measurements," Opt. Express 16, 6756-6767 (2008).

22. B. J. Rye, "Antenna parameters for incoherent backscatter heterodyne lidar," Appl. Opt. 18, 1390-1398 (1979).

23. M. Nakagami, "The $m$-distribution. A general formula of intensity distribution of rapid fading," in Statistical Methods in Radio Wave Propagation, W. C. Hoffman, ed. (Pergamon, 1960).

24. A. Belmonte and J. M. Kahn, "Performance of synchronous optical receivers using atmospheric compensation techniques," Opt. Express 16, 14151-14162 (2008).

25. R. J. Noll, "Zernike polynomials and atmospheric turbulence," J. Opt. Soc. Am. 66, 207-211 (1976).

26. J. W. Strohbehn, T. Wang, and J. P. Speck, "On the probability distribution of line-of-sight fluctuations of optical signals," Radio Sci. 10, 59-70 (1975).

27. J. W. Goodman, Speckle Phenomena in Optics. Theory and Applications ( Roberts \& Company, 2007).

28. R. F. Lutomirski and H. T. Yura, "Propagation of a finite optical beam in an inhomogeneous medium," Appl. Opt. 10, 1652-1658 (1971).

29. M. Born and E. Wolf, Principles of Optics (Cambridge University, 1999).

30. G. Dai, "Modal compensation of atmospheric turbulence with the use of Zernike polynomials and KarhunenLoève functions," J. Opt. Soc. Am. A 12, 2182-2193 (1995). 